\title{
46 \\ The IS-function: the most intelligent IT-tool
}

\author{
P.A.H.M. Mantelaers \\ Delft University of Technology, Department of Information Systems \\ P.O. Box 356, 2600 AJ Delft, The Netherlands \\ Tel:: +31 1527844 27, fax: +31 152786632 \\ E-mail: p.mantelaers@twi.tudelft.nl
}

\begin{abstract}
The information systems function (ISF) of an organization consists of all activities aiming at maintaining or extending the information (processing) capacity of that organization with the help of IT, and the means necessary to execute them. Attempts to improve the efficiency or the effectiveness of the ISF must address all its structural characteristics (Waterman, Peters \& Phillips, 1992). Each of these characteristics is an IT-tool and their optimal mix provides an organization with its most intelligent IT-tool. One of the often neglected characteristics is the organization of the ISF. A knowledge acquisition process was carried out to derive directives for the design of the ISF (Mantelaers, 1995).
\end{abstract}

\section{Keywords}

IS management, IS strategy, IS-function, organization design

\section{PROBLEM DEFINITION}

Every organization in the private and in the public sector ought to (re)consider the organization of its information function (OIF). From reports in professional periodicals it is shown that, in practice, many organizations reorganize their OIF. Applied research (e.g. Butler Cox, 1991) shows that many organizations expect to adapt their OIF in the near future, and have (repeatedly) done so. A more fundamental interest in this subject is apparent from articles in scientific journals (e.g. Swanson and Beath, 1989) and from conference reports.

Reasons for changing OIF are: problems with efficiency and/or effectiveness of the information function (IF) for the organization as a whole, dissatisfaction with IF of users/customers within the organization, the wish to follow trendy developments, and reorganization to bring 
about other changes. A more fundamental development is the fact that an increasing number of organizations is becoming aware of the strategic value of information. A conclusion to be drawn from this is that OIF deserves more attention. Conversely, thinking about OIF may increase the insight into the strategic value of information.

To improve the efficiency and effectiveness of the development of information systems, mainly new means are looked for, such as tools and methodologies. The question is whether these means have sufficiently stimulated, that systems become available within budget, time and specifications. For the management of operational information systems new methods are being developed as well, or it is often thought that the problems could be solved by outsourcing. In designing, building and managing systems, the results of these measures are not such that there is no reason to look for alternatives in order to solve IF problems: the reorganization of IF is one of those alternatives.

With the extension of the number of possible solutions (through the loss of oppressive boundary conditions from the available technology and the informaticians available on the labour market), the OIF problem has not become simpler or less important. Just as in designing information systems, in designing OIF it is always a matter of more than one solution. In principle, it is possible for various IF tasks to come to independent choices from all possible combinations of (de)centralization and (de)concentration. The answer to the question what would be the best alternative for a certain organization, will depend on specific circumstances. Moreover, it has to be taken into consideration that OIF cannot drastically be changed from one day to the next.

This paper summarizes my research (Mantelaers 1995) to provide a scientifically justified contribution to answer the following practical question: "How to design the most appropriate organization of the information function for an organization in a certain situation?"

First will be defined what is meant by (designing) OIF and what are the important characteristics of design methodologies with respect to this. Then the essence of the research is further pursued: what are design directives to be applied in terms of: design parameters (characteristics of OIF being appropriate to be influenced), contingency factors (factors influencing the setting up of the design parameters), and goal variables (the result to be aimed at by applying directives).

\section{DELINEATION OF CONCEPTS AND RESEARCH}

In this research an organization is defined as a whole of activities (with the relevant means) being executed to achieve a certain goal. In every organization these activities partly consist of information processing. The information function of an organization consists of all (combinations of) elementary information processing activities which can be distinguished within an organization and the means necessary to execute them. These means determine the capacity of the organization to provide itself or its environment with information. This capacity is called the information (processing) capacity (IC) of the organization at a certain point of time. Certain activities aim at improving this IC. The information systems function (ISF) of an organization consists of all activities aiming at maintaining or extending the IC of that organization with the help of IT, and the means necessary to execute them. The organization of ISF 
(OISF) has a number of structural characteristics: environment, objective, means, structure in the narrow sense, style of management, culture and skills.

Not IF as a whole, but only ISF is the subject of research. Its organization is considered within a certain organization to be defined beforehand. The external interorganizational organization problem is left out of consideration. A priori it is supposed to be known whether activities are executed inside or outside the organization. Another restriction is that only designing the organization of ISF is taken into consideration and not the transition processes resulting from this. The organization on behalf of which ISF has to be organized, is supposed to be known and fixed.

On the basis of the framework for methodologies for system development a framework has been developed, consisting of a number of characteristics which are of interest for the characterization and comparison of OISF methodologies. The following "ways" are part of it: way of thinking, way of modelling, way of working, way of diagnosing and designing, way of controlling, way of supporting, way of acting and foreknowledge. This framework has been applied in the further research.

\section{RESEARCH APPROACH}

In three ways it has been tried to gain insight into OISF methodologies. First, directives for the organization of ISF are gathered from literature. In doing so attention has been paid to general organization design theories (e.g. Douma and Schreuder, 1991), to (methodologies for) information strategy planning (e.g. Turner et al., 1988) and to research in the OITF field carried out by others (e.g. Bacon 1990). Besides, an analysis has been carried out of the reorganization of ISF as it has, in the past, actually taken place within two organizations.

The essence of this research consists of the knowledge acquisition process, carried out by having three OITF-experts given their opinion as to the way in which they would set up OISF for three organizations (Rabobank Nederland, Fokker Aircraft and the Ministry of VROM). Each experiment is first analyzed individually, using the framework for OISF methodologies. Thereupon the results have been compared together. In doing so, both similarities and differences per organization (between experts) and per expert (between organizations) have been looked for. In the rest of this paragraph the knowledge elicitation and knowledge analysis being carried out, are discussed.

From the methods available for knowledge elicitation a combination of observation and thinkaloud protocols has been chosen. Concurrent protocols and, additionally, retrospective protocols have been used, however, in both cases selectively: during the task implementation not every thought has been expressed by the experts and afterwards not everything has been explained. This method is completed with unstructured interviews.

Each experiment consisted of carrying out a consulting assignment in the field of OIF by an expert, and was allowed to take two days at the maximum, inclusive of the formulation of the advice. In these two days the expert could, in the presence of the researcher, have a number of interviews with functionaries of the organization in question. The whole has been registered on videotapes and audiotapes.

To be qualified for participation in the research, the experts had to dispose of a many years' experience in the field of OIF, to be acknowledged as experts by professionals in the 
field of study and to be prepared to reserve at least three times a period of two days for the research without being paid for it. The experts have been told explicitly that this research did not aim at evaluating their individual advice. The advice and the processes preceding it would only be used as means to come to conclusions about knowledge at a higher level.

With regard to the organizations a certain spread has been aimed at: both the private and the public sector had to be represented and within the private sector both physical and information-related transformation processes had to be involved. This has been chosen because of the assumption that these distinctions in particular would influence the design of OIF.

The protocol transcriptions (on the basis of conversations recorded on audio equipment and videotapes, and typed out literally) formed the starting point for the knowledge analysis. The aim was to isolate all knowledge that could be needed in solving the OISF problem. In analyzing the protocol transcriptions and the representation of the knowledge to be drawn from it, it had to be taken into consideration that each experiment had to be described in such a way that mutual comparison of the results of the 9 experiments was feasible. Next to that, the description ought to make possible that results could be used in practice by those who are considered to take decisions - make proposals - about the organization of ISF (external advisors or someone from within the organization).

In carrying out the knowledge analysis various methods have been tried out extensively. The methods being applied initially aimed at representing the information from the transcriptions in schemes. From the conviction that this way of representation demanded much energy, which was not compensated by profit in terms of insight, the choice was eventually a textual representation per "way". Also with this way of representation various experiments have been carried out before arriving at a definite choice. Choosing certain applied methods was based on considerations as: what is necessary and sufficient to achieve the goal of the research, how much time does an approach take, and how desirable is it to make the result of an analysis reducible without the necessity for someone to go back to the source material.

The individual reports of the nine experiments formed the basis of a mutual comparison, aiming at gaining insight into the influence of the difference in type of organization upon the method followed by an expert, and into the difference in methods applied by experts in one organization. When comparing the preference was given to a qualitative analysis. It is not a matter of determining in a statistically justified way how often certain ideas do occur to experts. It is a matter of determining whether an idea is useful for the objective of this research, even though it only occurs in one experiment.

The result of the research is phrased in terms of directives to be applied in practice for the design of the organization of ISF. At the same time they form a start for a design methodology for OIF.

For the subjects being most specific for the domain of OISF (the way of thinking and the way of diagnosing and designing (and with it also the way of modelling)), this led to the following five main directives: 
1. The design of OISF has to take place from a dynamic and open system vision.

2. In organizing the vision has to be assumed that:

- responsibilities ought to be integral;

- changing the organization means changing IF;

- organization is a continuous, reactive and pro-active activity;

- the functioning of the organization depends more on people than on structure.

3. The vision on coordination ought to be that it is necessary in order to outweigh the disadvantages of self government.

4. Organizing ISF requires denoting goal variables, contingency factors and design variables. The most important contingency factors are the structure of the organization and the information-related coherence between organization parts; the most important design variables are: responsibilities, organizational place of the execution of activities and the procedure structure.

5. The directives for the organization of ITF can be summarized as follows: make line management responsible for the internal working and the external tuning, and integrate the exeeution in the-primary process.

The five directives (for demands to be made upon OISF designers) in the sphere of foreknowledge, way of supporting, way of acting, way of working and way of controlling are mainly based on the experiments and are more general in character:

- Applying practical experience (whether or not own) in the right way is essential; (fore)knowledge is a useful addition here.

- A framework (based on experience) for the problem field is the best way of supporting; a good memory is an important aid.

- Flexibility as to the way of acting is essential for the acceptance of the designer by the organization, and so for his effectiveness.

- The main line in the way of working is to be characterized as a top-down search for signals being connected with OISF, where getting acquainted, analyzing and designing strongly overlap.

- Using appropriate techniques influences the productive use of the time available for intelligence.

A noticeable fact is that in this research information from three sources (at first sight noncoherent and different working methods of experts, divergent observations from the historical research and research of others) being directed to different points for special attention could be organized in a relatively small number of coherent directives. On the basis of this result further research can be directed towards the development of a methodology for the organization of the information (systems) function.

\section{REFERENCES}

Butler Cox (1991) Managing the Devolution of Systems Responsibilities, Research Report 81, Butler Cox Foundation.

Bacon, C.J. (1990) Organizational Principles of Systems Decentralization. Journal of Information Technology, 5, 84-93. 
Douma, S. \& H. Schreuder (1992) Economic Approaches to Organizations. Prentice Hall, Englewood Cliffs.

Mantelaers, P.A.H.M. (1995) Information Capacity Engineering. Doctoral thesis (in Dutch), Delft University of Technology, Delft.

Swanson, E.B. \& C.M. Beath (1989) Reconstructing the systems development organization. MIS-Quarterly, 13, 3, 293-305.

Turner, W.S., R.P. Langerhorst, G.F. Hice, H.B. Eilers \& A.A. Uijttenbroek (1988) System Development Methodology. Pandata B.V., Rijswijk.

Waterman, R.H., Jr., T.J. Peters \& J.R. Phillips (1992) The 7-S framework, in The Strategy Process (eds. H. Mintzberg and J.B. Quinn), Prentice-Hall, Englewood Cliffs.

\section{BIOGRAPHY}

Paul Mantelaers is senior lecturer at Delft University of Technology. He holds a degree in Business Administration from Tilburg University and completed recently his doctoral thesis on the design of the organization of the IS-function. He teaches in Information Management and Information Systems Design. His research interests include organizational and economic aspects of information management and transnational systems development. 